Effect of pinning on the response of superconducting strips to an external pulsed current

This content has been downloaded from IOPscience. Please scroll down to see the full text.

2015 Supercond. Sci. Technol. 28025004

(http://iopscience.iop.org/0953-2048/28/2/025004)

View the table of contents for this issue, or go to the journal homepage for more

Download details:

IP Address: 146.175.11.111

This content was downloaded on 24/07/2015 at 10:19

Please note that terms and conditions apply. 


\title{
Effect of pinning on the response of superconducting strips to an external pulsed current
}

\author{
G Berdiyorov ${ }^{1,2}$, K Harrabi $^{3}$, J P Maneval ${ }^{4}$ and F M Peeters ${ }^{5}$ \\ ${ }^{1}$ Department of Physics, Loughborough University, Leicestershire, LE11 3TU, UK \\ ${ }^{2}$ Qatar Environment and Energy Research Institute, Doha, Qatar \\ ${ }^{3}$ Physics Department, King Fahd University of Petroleum and Minerals, 31261 Dhahran, Saudi Arabia \\ ${ }^{4}$ Laboratoire Pierre Aigrain, ENS 24 rue Lhomond, Paris F-75231, France \\ ${ }^{5}$ Departement Fysica, Universiteit Antwerpen, Groenenborgerlaan 171, B-2020 Antwerpen, Belgium \\ E-mail: francois.peeters@uanwerpen.be
}

Received 18 July 2014, revised 15 October 2014

Accepted for publication 22 October 2014

Published 31 December 2014

\begin{abstract}
Using the anisotropic time-dependent Ginzburg-Landau theory we study the effect of ordered and disordered pinning on the time response of superconducting strips to an external current that switched on abruptly. The pinning centers result in a considerable delay of the response time of the system to such abrupt switching on of the current, whereas the output voltage is always larger when pinning is present. The resistive state in both cases are characterized either by dynamically stable phase-slip centers/lines or expanding in-time hot-spots, which are the main mechanisms for dissipation in current-carrying superconductors. We find that hot-spots are always initiated by the phase-slip state. However, the range of the applied current for the phase-slip state increases significantly when pinning is introduced. Qualitative changes are observed in the dynamics of the superconducting condensate in the presence of pinning.
\end{abstract}

Keywords: superconductor, phase slip, hot spot

(Some figures may appear in colour only in the online journal)

\section{Introduction}

Phase-slip centers (PSCs) are one of the main mechanisms for dissipation in narrow current-carrying superconducting thin films, resulting in the formation of pronounced steps in both voltage versus temperature [1] and current-voltage characteristics $[2,3]$. In the phase-slip state the superconducting order parameter oscillates in time allowing the phase to relax by quanta of $2 \pi[4,5]$. For superconducting samples with lateral dimensions larger than the superconducting coherence length $\xi$, PSCs are converted into phase-slip lines with almost uniform suppression of the order parameter along the sample [6]. The oscillations of the order parameter can also occur in the form of propagating waves creating a $2 \pi$ phase difference across the sample, which are called kinematic vortices [7-11]. These vortices move with velocity [9] $v_{\mathrm{kv}} \approx 10^{5} \mathrm{~m} \mathrm{~s}^{-1}$, which is much larger than the maximal measured speed of Abrikosov vortices [12] $v_{\mathrm{av}} \approx 10^{3} \mathrm{~m} \mathrm{~s}^{-1}$. Because of their very high velocity kinematic vortices do not retain their circular structure [7, 8, 13].

Another mechanism for the current-induced resistive state is the formation of hot spots, where the normal state is realized due to the local increase of temperature [14]. It is believed that the phase-slip regime occurs close to the superconducting transition temperature [1,3], and that the hot spot mechanism dominates at lower temperatures [14]. However, recent experimental studies implementing the pulsed current technique revealed that hot spots never form unless PSCs have first been nucleated [15-18]. Numerical simulations within the time-dependent Ginzburg-Landau (TDGL) theory confirmed that the hot spot regime is always initiated by the phase-slip mechanism [19].

In this work we investigate the effect of pinning of the response of a superconducting strip to external current, which is switched on abruptly, using the TDGL theory. We are motivated by the fact that the response of any superconductor 


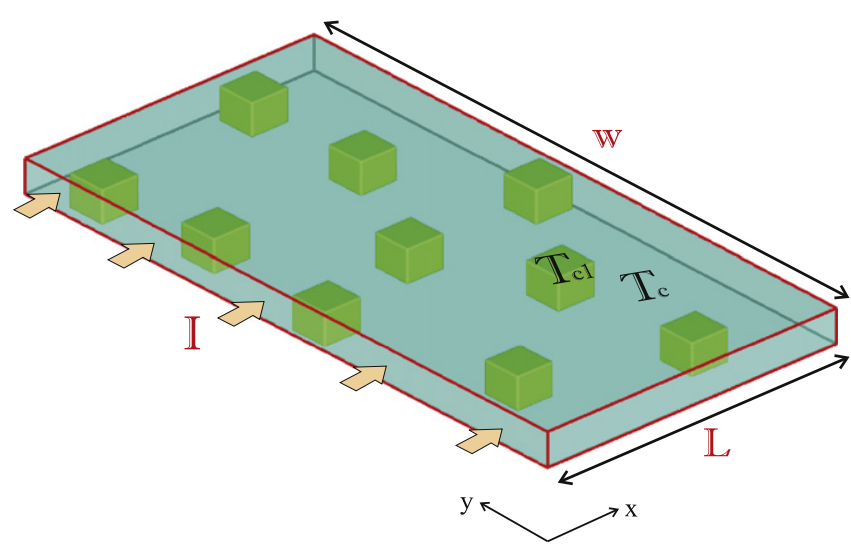

Figure 1. The model system: a current-carrying superconducting strip (dimensions $w \times L \times d$ ) with weakly superconducting inclusions (of size $a$ ) with critical temperature $T_{\mathrm{c} 1}<T_{\mathrm{c}}$.

to external electric and magnetic fields is strongly affected by the disorder in the system and by edge imperfections [20], which are inevitable in real superconductors. For example, the critical field for penetration of vortices in type-II superconductors is strongly reduced when introducing edge defects, resulting in qualitative changes in the magnetization curves of the system (see, e.g., [21]). The disorder also results in multiple reentrant transitions between the superconducting and normal states by increasing the applied current (see, e.g., [17]). In current-carrying superconductors the inhomogeneities strongly affect the current distribution in the system, leading to qualitative changes in the dynamics of the superconducting condensate [7]. Our numerical simulations show that the response time of the system to the external current decreases considerably when introducing pinning due to the reduced barrier for the nucleation of superconducting vortices/antivortices. Motion of these vortices/antivortices is dynamically stable in time and are responsible for the resistive state of the system at low current values. At larger currents the fast moving vortices initiate the formation of hot-spots, which expand in time until the whole system switches into the normal state.

The manuscript is organized as follows. Section 2 represents our theoretical approach. Section 3 is about the current-voltage characteristics of the sample without and with the ordered and random pinning. In section 4 we study response of our system to abrupt switching on of the external current by calculating the voltage versus time characteristics of the sample. Our findings are summarized in section 5 .

\section{Theoretical formalism}

As model system we consider a superconducting strip with lateral dimensions $L$ and $w$ of the computational unit cell. The pinning is introduced as square inclusions (size $a$ ) of another superconducting material with smaller critical temperature $T_{\mathrm{c} 1}<T_{\mathrm{c}}$ (see figure 1). The thickness $d$ is much smaller than the coherence length $\xi$ and the penetration depth. Current is applied along the $x$-direction. For this system we solved numerically the following TDGL equations:

$$
\begin{aligned}
& \frac{u}{\sqrt{1+\gamma^{2}|\psi|^{2}}}\left(\frac{\partial}{\partial t}+\frac{\gamma^{2}}{2} \frac{\partial|\psi|^{2}}{\partial t}\right) \psi \\
& =(\nabla-i \mathbf{A})^{2} \psi+\left(f(\vec{r})-|\psi|^{2}\right) \psi, \\
& \frac{\partial \mathbf{A}}{\partial t}=\operatorname{Re}\left[\psi^{*}(-i \nabla-\mathbf{A}) \psi\right]-\kappa^{2} \operatorname{rot} \operatorname{rot} \mathbf{A},
\end{aligned}
$$

where the parameter $f(\vec{r})$ (see [23] for its definition) accounts for the $T_{\mathrm{c}}$-nonhomogeneity in the system: for $f(\vec{r})<1$ (i.e., $T_{\mathrm{c} 1}<T_{\mathrm{c}}$ ) superconductivity is strongly suppressed inside the defect, consequently it attracts the flux domains, whereas in the case of $f(\vec{r})>1$ (i.e., $T_{\mathrm{c} 1}>T_{\mathrm{c}}$ ) the defect interacts with the flux repulsively. The equation (1) with the inhomogeneity coefficient $f(\vec{r})$ which is valid at $T=0$, has already been used to describe both static [22] and dynamic [23] properties of type-II superconductors with weakly superconducting inclusions. In equations (1) and (2) we express the length in units of the coherence length $\xi$ and the vector potential is scaled to $\Phi_{0} /(2 \pi \xi)$ (where $\Phi_{0}$ is the magnetic flux quantum). Time is in units of the Ginzburg-Landau relaxation time $t_{\mathrm{GL}}=4 \pi \lambda^{2} / c^{2} \rho_{n}$ ( $\rho_{n}$ is the normal-state resistivity), the voltage, which is calculated as $V=-\int \partial \mathbf{A} / \partial t \cdot \mathbf{d l}$, is in units of $V_{0}=c \Phi_{0} \rho_{n} / 8 \pi^{2} \lambda \xi$ and the current density is measured in $j_{0}=c \Phi_{0} / 8 \pi^{2} \lambda^{2} \xi$. $\psi_{0}=4 k_{B} T_{c} u^{1 / 2} / \pi$ is the value of the order parameter at $T=0$. The parameters $u$ and $\gamma$, which are measures of the different relaxation times, are taken as $u=5.79$ and $\gamma=10$ [24]. The numerical results are obtained for the GL parameter $\kappa=\lambda / \xi=10$. The infinite strip is implemented through the periodic boundary conditions $\psi(x, y)=\psi(x+L, y)$ and $\mathbf{A}(x, y)=\mathbf{A}(x+L, y)$ in the $x$-direction. The superconducting-vacuum boundary condition $\left.(\nabla-i \mathbf{A}) \psi\right|_{y=0, w}$ is used in the $y$-direction. A current is applied by taking the following boundary condition for the vector potential in the $y$ direction, $\operatorname{rot} \mathbf{A l}_{z}(y=0, w)=H \pm H_{I}$, where $H$ is the applied magnetic field (zero in our case) and $H_{I}=2 \pi I / c$ is the magnetic field induced by the current $I$. Note that in equations (1) and (2) the screening of the magnetic field is neglected, which is valid only for very thin superconductors or for samples that extend infinitely in the $z$-direction. These coupled nonlinear differential equations are solved self-consistently in a zero electrostatic potential gauge [25], which was recently shown to be very effective in studying the dynamics of superconducting vortices [13].

\section{I- V characteristics}

\subsection{Pinning-free case}

As a representative example, we consider a superconducting strip with dimensions $L=32 \xi$ and $w=64 \xi$ in the presence of regular (a square array) and random pinning. As a reference for our further analysis, we first demonstrate the properties of a pinning-free sample by constructing the time averaged voltage versus applied current $(I V)$ characteristics of the 

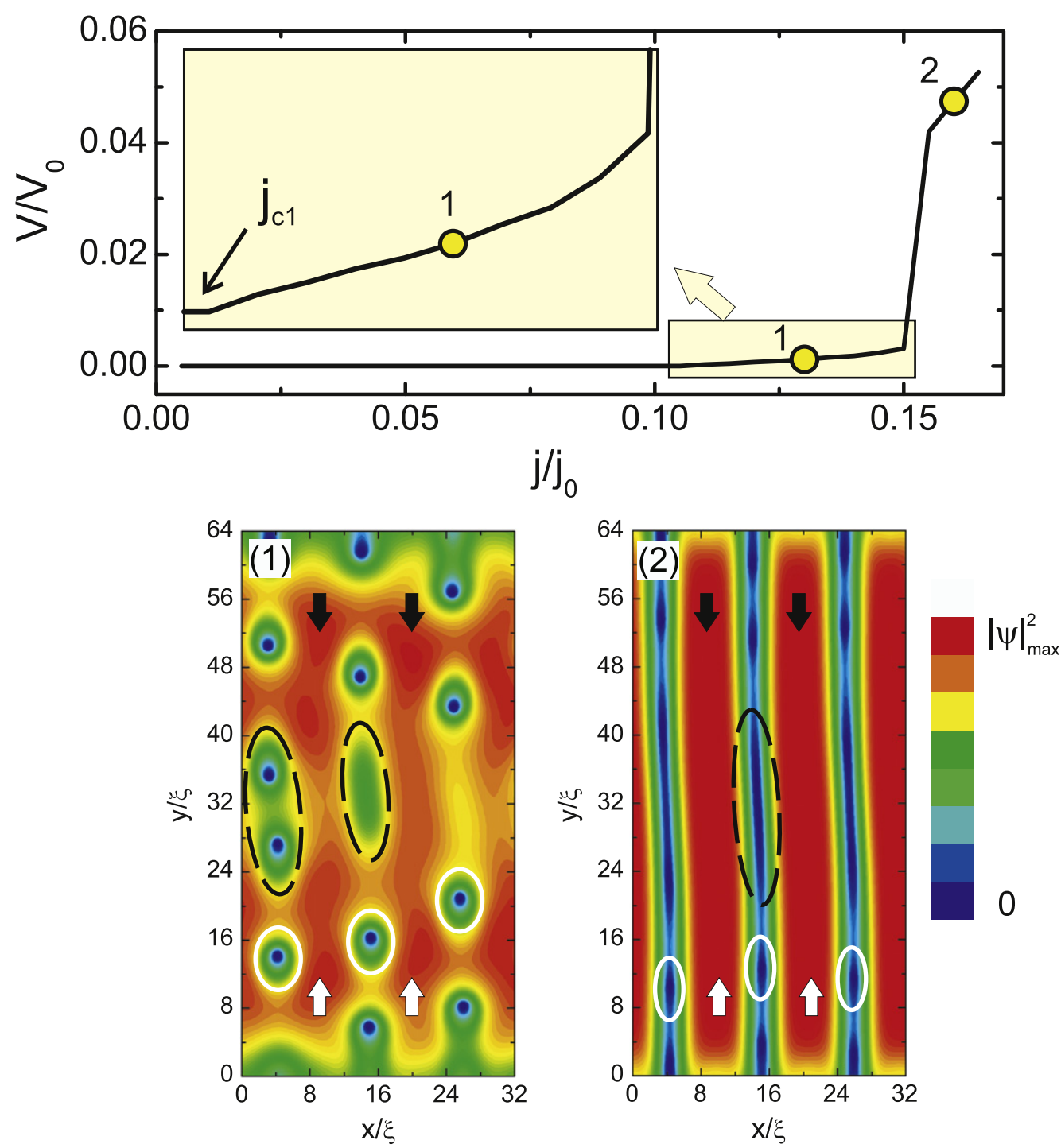

Figure 2. Time averaged voltage versus current density $(I-V)$ characteristics of the sample with width $w=64 \xi$ and $L=32 \xi$. Inset in the main panel shows the lower part of the $I-V$ curve. Panels 1 and 2 show snapshots of the Cooper-pair density $|\psi|^{2}$ (red/blue corresponds to largest/zero $|\psi|^{2}$ ) for current values indicated on the $I-V$ curves. Black/white arrows indicate the direction of motion for vortices/antivortices and white ellipses highlight some of the antivortices. Black ellipses show the annihilation region of a vortex-antivortex pair.

system, which is shown in figure 2 together with snapshots of the Cooper-pair density (panels 1 and 2). With increasing applied current, zero resistance of the sample is maintained up to a threshold current density $j_{\mathrm{c} 1}=0.11 j_{0}$, above which the system switches into a resistive state. At low currents the resistive state is characterized by the periodic nucleation of slow moving Abrikosov vortex-antivortex pairs. Since no external magnetic field is present, those vortices are created periodically at the opposite edges of the sample and annihilate in the middle of the sample (highlighted by dashed ellipses in inset 1). However, the voltage signal due to Abrikosov vortices is much smaller than the one generated by the fast moving vortices (compare points 1 and 2 in figure 2). This signal increases monotonically with applied current indicating a larger nucleation rate of vortex-antivortex pairs. By further increasing the applied current, the triangular vortex lattice gets distorted and a rectangular lattice of vortices is observed (see panel 2) [10, 13]. At this stage the vortices are deformed and resemble the structure of fast-moving (kinematic) vortices reported earlier [7, 8]. The distortion is more pronounced in the middle of the sample during the annihilation of vortexantivortex pairs, where the speed of the vortices are largest [8]. This transition from a slow to fast moving vortex state results in a noticeable jump in the $I-V$ curve (see point 2 ). With further increase of the applied current more channels with fast moving vortices appear in the system (not shown here) before the entire sample switches into the normal state. Thus, depending on the applied current the resistive state is characterized either by slow moving Abrikosov vortices or fast moving vortices (or phase-slip lines).

\subsection{Square array of pinning centers}

Next, we introduce a square lattice of pinning centers and study its effect on the critical parameters of the sample. As an 

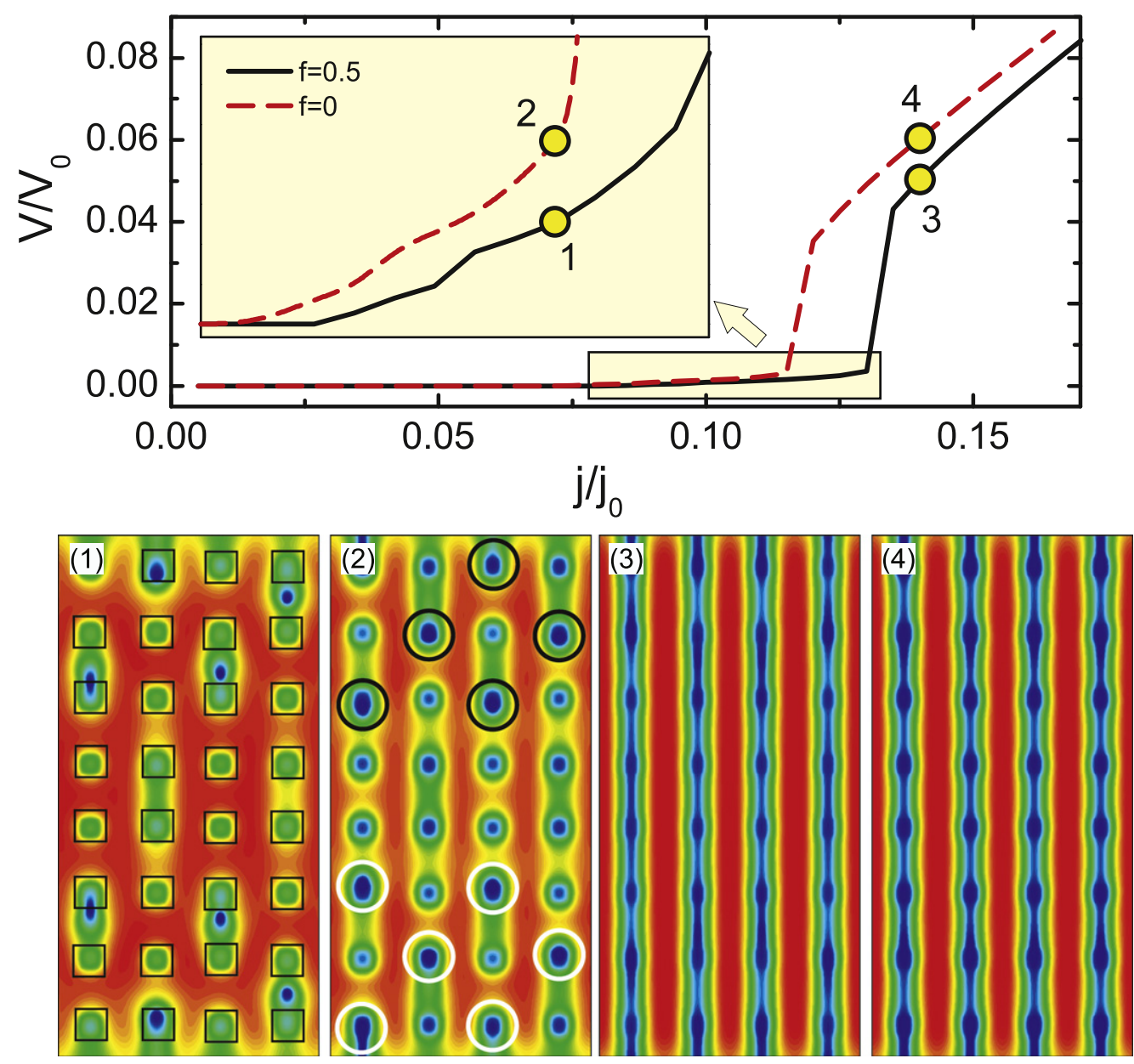

Figure 3. $I-V$ characteristics of the sample with width $w=64 \xi$ and $L=32 \xi$ in the presence of a square array of pinning center of size $a=2 \xi$ and period $d=8 \xi$ for the inhomogeneity coefficient of $f(\vec{r})=0.5$ (solid black curve) and $f(\vec{r})=0$ (dashed red curve). Inset in the main panel shows the lower part of the $I-V$ curve. Panels 1-4 show snapshots of $|\psi|^{2}$ for current values indicated on the $I-V$ curves. Location of the pinning centers are shown by black squares in panel 1. Black/white circles in panel 2 highlight the position of vortices/ antivortices.

example, we consider a square array of pinning centers of size $a=2 \xi$ and period $d=8 \xi$ for two different values of the inhomogeneity coefficient $f(\vec{r})$. The dimensions of the sample are the same as in figure 2. Figure 3 shows the $I V$ characteristics of the sample with $4 \times 8$ pinning centers for two values of $f(\vec{r})$. It is seen from this figure that the critical current is significantly reduced by introducing pinning $\left(j_{\mathrm{c} 1}=0.08 j_{0}\right)$ due to the lowering of the penetration barrier for the vortices by the pinning centers near the sample edge [21]. $j_{\mathrm{c} 1}$ decreases further with increasing pinning strength, i.e. the inhomogeneity coefficient $f(\vec{r})$ (compare solid black and dashed red curves in figure 3). Another mechanism for the reduction of the critical current could be the attraction of vortices/antivortices by the pinning centers, resulting in their easier penetration. However, the resistive state is still characterized by the periodic nucleation and annihilation of either slow (panels 1 and 2) or fast moving (panels 3 and 4) vortexantivortex pairs. Notice that, although the inhomogeneity parameter $f$ changes stepwise at the defect interface, the order parameter changes smoothly at the interface due to the proximity effect $[23,24]$. Slowly moving vortices/antivortices attempt to form a triangular lattice (see black/white circles in panel 2) to minimize their mutual repulsion. At larger currents this triangular lattice gets distorted and a square lattice of vortices is observed (panels 3 and 4). A large jump in the output voltage is observed due to this structural transition. The number of phase-slip lines are determined by the number of columns of pinning centers.

\subsection{Random pinning}

Figure 4 shows the $I-V$ characteristics of the system for two values of $f(\vec{r})$ when random pinning is introduced (see back squares in panel 1). The size of the pinning centers and their density (i.e. the number of pinning centers) are the same as in the case of regular pinning (see figure 3). For this particular distribution of the pinning centers, the critical current becomes slightly smaller $\left(j_{\mathrm{c} 1}=0.07 j_{0}\right)$ than the one for the ordered pinning $\left(j_{\mathrm{c} 1}=0.08 j_{0}\right)$. This is because some of the pinning centers are located close to the edges, resulting in the local reduction of the surface barrier. In addition, they form an easy channel for the motion of superconducting vortices facilitating their transport through the system (see panels 1 

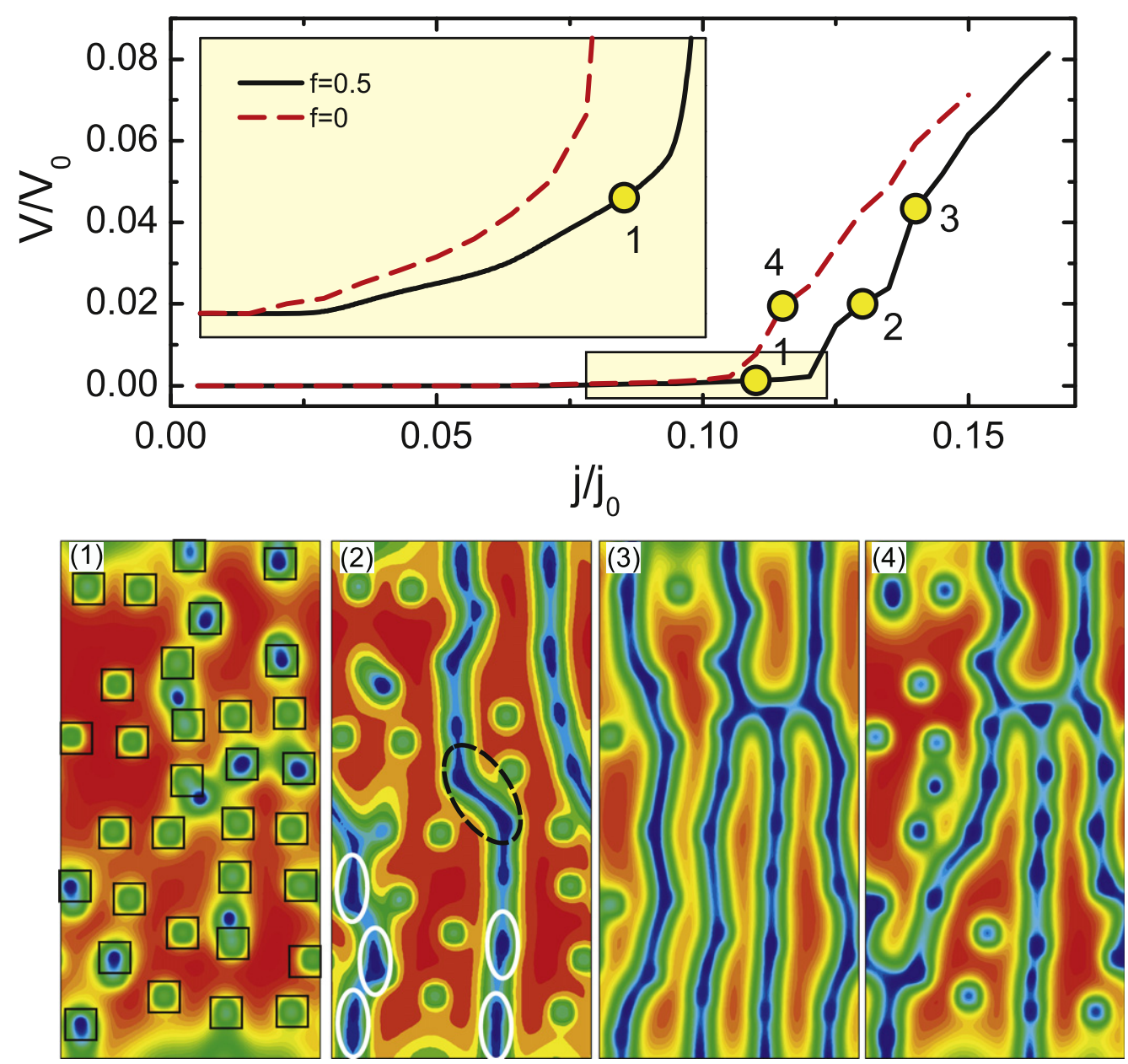

Figure 4. $I-V$ characteristics of the sample with width $w=64 \xi$ and $L=32 \xi$ in the presence of random pinning centers of size $a=2 \xi$ for the inhomogeneity coefficient of $f(\vec{r})=0.5$ (solid black curve) and $f(\vec{r})=0$ (dashed red curve). Inset in the main panel shows the lower part of the $I-V$ curve. Panels 1-4 show snapshots of $|\psi|^{2}$ for current values indicated on the $I-V$ curves. Location of the pinning centers are shown by black squares in panel 1. Locations of the antivortices are highlighted by white ellipses and one of the annihilation points is indicated by the dashed-black ellipse.

and 2). The resistive state is still characterized by the nucleation/annihilation of slow moving Abrikosov vortices (panel 1) and fast moving kinematic vortices (panels 2-4). However, due to the randomness in the distribution of the pinning centers, the number of channels (i.e. PSCs) for vortices and antivortices are not the same: for this particular distribution of the pinning centers the number of antivortex channels is always larger than the one for the vortices (panels 3 and 4). Depending on the arrangement of the pinning centers, one can have the opposite case, i.e., more channels for the vortices than the ones for the antivortices.

\section{Voltage-time characteristics}

In what follows, we study the time delay of our system to an abrupt switching on of applied current at zero external magnetic field, by constructing voltage versus time characteristics of the sample. We started our simulations from the Meissner state (i.e., $|\psi|=1$ ) and applied a dc current pulse with duration $5000 t_{0}$ at $t=10 t_{0}$. During that time period, we recorded the voltage versus time $(V(t))$ characteristics of the system together with the evolution of the superconducting state. To show the properties of the resistive state in response to the current we plot in figure 5 (a) $V(t)$ curves of the pinningfree sample with dimensions $L=32 \xi$ and $w=64 \xi$ for different values of the applied current density. Snapshots of the Cooper-pair density are also presented there to show the time evolution of the superconducting condensate (see panels 1-4). At smaller currents the resistive state is characterized by slow motion of vortices and antivortices (as shown in panel 1 of figure 2) and their annihilation results in well defined peaks in the $V(t)$ curves (not shown here). $V(t)$ curves show periodic oscillations as has been reported in previous works [26-30]. With increasing applied current, phase slip lines are formed (panels 1 and 2 in figure 5) and the output voltage increases considerably (see curve I in figure 5(a)). The average voltage signal saturates to a certain value with small oscillations, which are due to the penetration and/or annihilation of fast moving vortices. With further increasing the applied current the nucleation rate of vortex-antivortex pairs, as well as the number of phase-slip lines increases (see panel 2 in figure 5) 

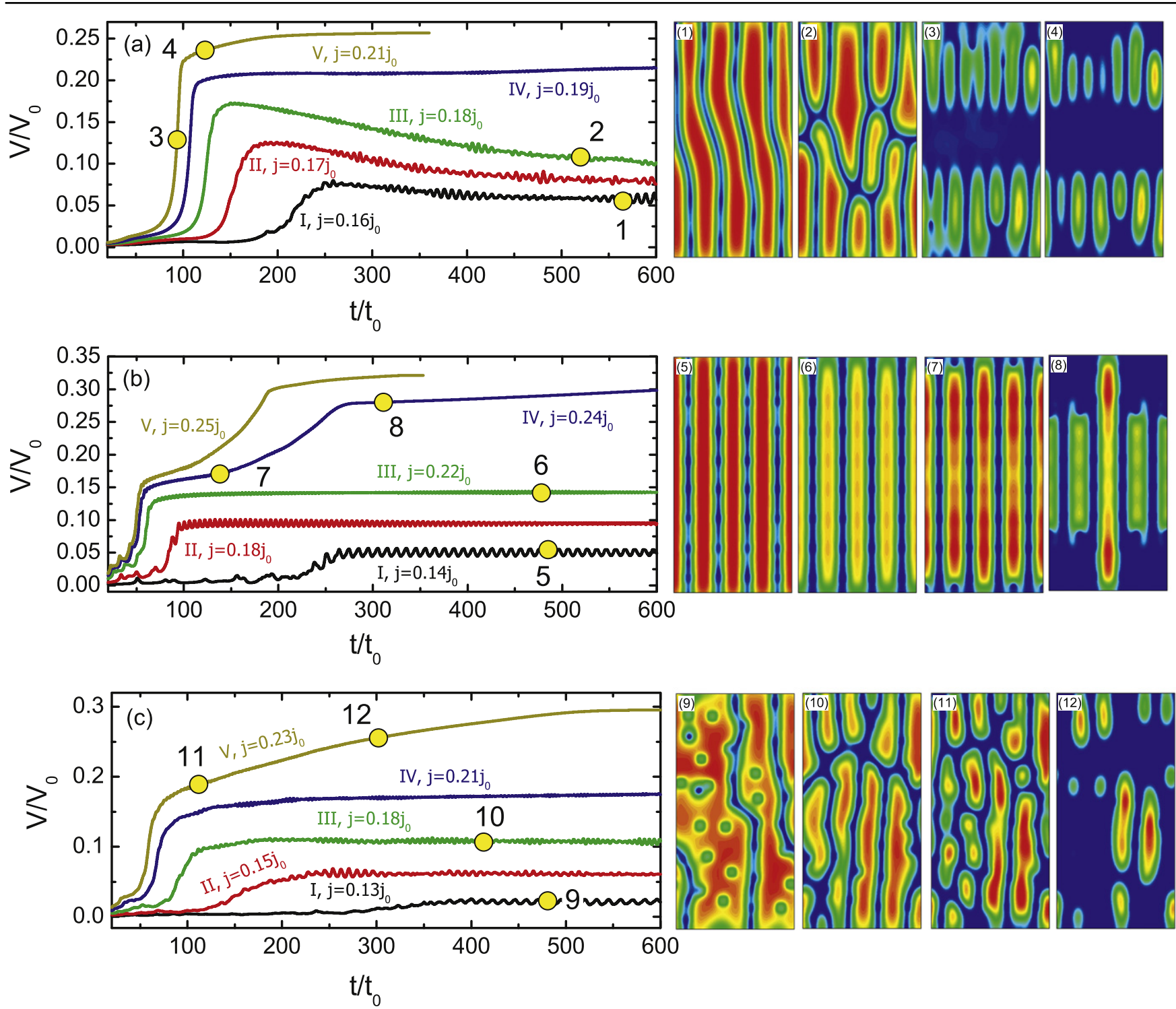

Figure 5. Voltage versus time $(V-t)$ curves of the sample with dimensions $L=32 \xi$ and $w=64 \xi$ without pinning (a), with regular (square array) pinning (b) and with random pinning (c) for different values of the applied current pulse $j \delta\left(t-10 t_{0}\right)$. The voltage is shown for $t>10 t_{0}$. The size of the pinning centers is $a=2 \xi$ and the inhomogeneity coefficient is $f(\vec{r})=0.5$. Panels $1-12$ show snapshots of the Cooper-pair density $|\psi|^{2}$ at time indicated on the $V(t)$ curves (for the used scale see figure 2).

resulting in an increase of the output voltage (curve II in figure 5(a)). At these high values of the applied current, the voltage reaches a maximum just before the formation of phase-slip lines (see curves II and III in figure 5(a)), which we relate to nonequilibrium effects at the initial stage of the resistive state formation. However, the voltage curves saturate when the phase-slip state is established (see panel 2). Notice that the phase slip lines are not necessarily straight and parallel. With further increasing the applied current, the number and velocity of vortex-antivortex pairs increases and local hot spots areas appear, where the density of Cooper-pairs reduces to zero. Those hot spots are created near the sample edges and in the middle of the sample (see panel 3), where the local velocity of the vortices become maximal (see figure 8 of [8]). Once created, the size of the hot spots increases in time (see panel 4) and the entire system becomes normal. Thus, the hot spots are unstable in time: they expand until the entire system makes a transition to the normal state.

Figure 5(b) shows the $V(t)$ curves of the sample with a square array of pinning centers for $f(\vec{r})=0.5$ and for different values of the applied current.The presence of the defects changes the voltage curve of the sample considerably: (i) transition to the phase-slip state occurs at smaller currents due to the reduced barrier for nucleation of superconducting vortices [21] (for example for the pinning free sample the transition to the resistive state occurs at $j=0.155 j_{0}$, whereas for the sample with square pinning arrays this current equals to $j=0.14 j_{0}$ ); (ii) the delay time in the response of the system to the applied current pulse becomes smaller (compare curves III in figures 5(a) and (b)), which we again relate to the reduction of the surface barrier; (iii) the output voltage saturates faster as compared to the pinning-free sample and no 

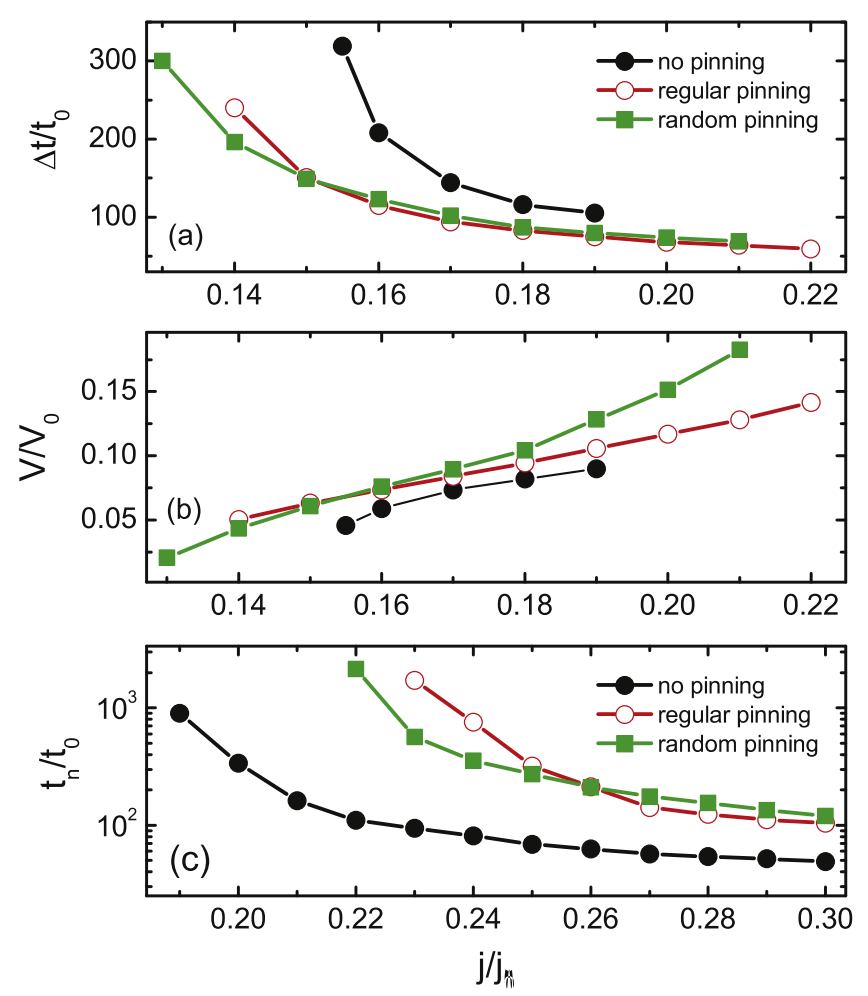

Figure 6. (a), (b) Delay time $\Delta t$ (a) and saturated voltage (b) as a function of applied current when the system switches to the phaseslip state. (c) Normal state transition time $\Delta t_{\mathrm{n}}$ as a function of applied current. The dimensions of the system and the pinning centers are the same as in figure 5 .

maxima in the $V(t)$ curve is observed at the initial stage of the resistive state formation (compare curves III in figures 5(a) and (b)); and (iv) the hot spots start nucleating at the sample edges (see panel 8), whereas in the pinning-free sample the hot spots dominate in the middle of the sample (see panel 4). In addition longer time is required for the normal state transition of the whole system (compare curve $\mathrm{V}$ in figure 5(a) and curve IV in figure 5(b)).

Finally, figure $5(\mathrm{c})$ shows the $V(t)$ curves of the sample with random distribution of the pinning centers. The dimensions of the sample and size, strength and density of the pinning centers are the same as in figure 5(b). As in the case of regular pinning, random pinning also reduces the response time of the system as compared to the reference sample (compare curves obtained for $j=0.18 j_{0}$ in figures 5(a)-(c)). However, in contrast to regular pinning, we observe irregularities in the voltage curves which results from the disordered motion of superconducting vortices (see panels 9 and 10). Hot spots also appear randomly both near the edges of the sample and in its interior (panels 11 and 12). In all three cases, the resistive state is characterized either by slow or fast moving vortices and the latter initiate the formation of the hot spots, which are not stable in time and results in a complete destruction of superconductivity. Similar behavior of the sample is observed for larger strength of the pinning centers.

To summarize our findings about the effect of pinning on the response of superconducting strips to an abrupt switching on of external current, we plotted in figure 6(a) the delay time in the formation of the phase-slip state as a function of applied current for the samples with and without pinning. The delay time is calculated as the difference between the switching time of the current pulse $\left(t=10 t_{0}\right)$ and the characteristic time $t^{*}$, when the output voltage equals half of the saturated voltage at larger times. It is seen from this figure that for all samples the delay time monotonically decreases with increasing applied current. However, the delay time is reduced considerably when pinning is introduced. This effect becomes more pronounced at smaller current, where the delay time increases faster for the pinning-free sample. As we have discussed above, the reason for such reduction of the delay time is the reduced penetration barrier for the superconducting vortices. The difference between the delay times for samples with regular and irregular pinning is very small: it is slightly larger for random pinning. However, at smaller current the delay time becomes larger in the case of ordered pinning centers. Figure 6(b) shows the saturated voltage (averaged over a time interval larger than the characteristic oscillations of the $V(t)$ curve) as a function of applied current in the phaseslip state. Smallest voltage is observed for the pinning-free sample (filled black dots) and the largest signal is obtained in the case of irregular pinning (green squares). Such an increase of the output voltage indicates a larger velocity of the kinematic vortices. The latter effect is due to the fact that the current density becomes larger between the pinning centers due to the current crowding [29]. The output voltage reduces when random pinning is replaced by a periodic one (compare solid squares and open circles). This is due to the disorder to order transition in the dynamics of the superconducting vortices [31]. Note also that the current range where the phaseslip state is realized is much smaller for the pinning free sample as compared to the other cases. Figure 6(c) shows the transition time $t_{\mathrm{n}}$ to the normal state, i.e., the time when the whole system passes to the normal state, as a function of the applied current. Contrary to the case of the phases-slip state, now the normal state transition time is much smaller for the pinning-free sample (solid circles). This is due to the fact that the pinning centers create an effective path for the vortices to move, thus preserving the rest of the superconducting condensate from destruction. Only a small difference in $t_{\mathrm{n}}$ is noted when two different types of pinning are introduced (open circles and filled squares), which becomes more pronounced at smaller currents.

\section{Conclusions}

Using the anisotropic TDGL theory, we studied the effect of ordered and disordered pinning on the response of a superconducting strip to applied current. We found that pinning considerably reduces the resistive state transition current, which we relate to the reduced edge barrier for the superconducting vortices. For the same reason, the delay time of the system to the abrupt switching on of the current is also reduced by pinning. Qualitative changes are observed in the dynamics of the superconducting condensate (and consequently in the output signal) when pinning is included. 
However, regardless of the presence of pinning, the resistive state is characterized by: (i) a flux-flow state at smaller current, (ii) the phase-slip state (or fast moving vortices) at intermediate values of the current or, (iii) the hot spot state at larger currents. The first two states are found to be stable in time, whereas the hot spot, fed with a constant current, evolves into a complete transition of the sample into the normal state. The normal state transition time is found to be smaller for the pinning-free sample.

\section{Acknowledgments}

This work was supported by EU Marie Curie (Project No: 253057), the Flemish Science Foundation (FWO-Vl) and King Fahd University of Petroleum and Minerals, Saudi Arabia, under the IN131034 DSR project.

\section{References}

[1] Webb W W and Warburton R J 1968 Phys. Rev. Lett. 20461

[2] Meyer J D 1973 Appl. Phys. 2303

[3] Skocpol W J, Beasley M R and Tinkham M 1974 J. Low Temp. Phys. 16145

[4] Tinkham M 1996 Introduction to Superconductivity 2nd edn (Singapore: McGraw-Hill) chapter 11

[5] Ivlev B I and Kopnin N B 1984 Usp. Fiz. Nauk 142435 Ivlev B I and Kopnin N B 1984 Sov. Phys.-Usp. 27206

[6] Weber A and Kramer L 1991 J. Low Temp. Phys. 84289

[7] Andronov A, Gordion I, Kurin V, Nefedov I and Shereshevsky I 1993 Physica C 213193

[8] Berdiyorov G R, Milošević M V and Peeters F M 2009 Phys. Rev. B 79184506

[9] Sivakov A G, Glukhov A M, Omelyanchouk A N, Koval Y, Muller P and Ustinov A V 2003 Phys. Rev. Lett. 91267001

[10] Silhanek A V, Milošević M V M V, Kramer R B G, Berdiyorov G R, van de Vondel J, Luccas R F, Puig T, Peeters F M and Moshchalkov V V 2010 Phys. Rev. Lett. 104017001
[11] Bulaevskii L N, Graf M J and Kogan V G 2012 Phys. Rev. B 85014505

[12] Tinkham M 1979 J. Low Temp. Phys. 3517

[13] Vodolazov D Y and Peeters F M 2007 Phys. Rev. B 76014521

[14] Skocpol W J, Beasley M R and Tinkham M 1974 J. Appl. Phys. 454054

[15] Ladan F-R, Harrabi Kh, Rosticher M, Villard C, Mathieu P and Maneval J-P 2008 J. Low Temp. Phys. 153103

[16] Harrabi K, Ladan F R, Maneval J-P, Lam V D, Hamet J-F, Villégier J-P and Bland R W 2009 J. Low Temp. Phys. 15736

[17] Harrabi K 2013 J. Supercond. Nov. Magn. 261865

[18] Maneval J P, Harrabi K, Chibane F, Rosticher M, Ladan F R and Mathieu P 2013 IEEE Trans. Appl. Supercond. 232200604

[19] Berdiyorov G, Harrabi K, Oktasendra F, Gasmi K, Mansour A I, Maneval J P and Peeters F M 2014 Phys. Rev. B 90134505

[20] de Gennes P G 1989 Superconductivity of Metals and Alloys (Redwood City, CA: Addison-Wesley)

[21] Vodolazov D Y, Maksimov I L and Brandt E H 2003 Physica C 384211

[22] Liu C Y, Berdiyorov G R and Milosevic M V 2011 Phys. Rev. B 83104524

[23] Berdiyorov G R, de A R, Romaguera C, Milošević M V, Doria M M, Covaci L and Peeters F M 2012 Eur. Phys. J. B 85130

[24] Kramer L and Watts-Tobin R J 1978 Phys. Rev. Lett. 401041 Watts-Tobin R J, Krähenbühl Y and Kramer L 1981 J. Low Temp. Phys. 42459

[25] Winiecki T and Adams C S 2002 J. Comput. Phys. 179127

[26] Berdiyorov G R, Elmurodov A K, Peeters F M and Vodolazov D Y 2009 Phys. Rev. B 79174506

[27] Berdiyorov G R, Milošević M V and Peeters F M 2009 Phys. Rev. B 80214509

[28] Berdiyorov G R, Chao X H, Peeters F M, Wang H B, Moshchalkov V V and Zhu B Y 2012 Phys. Rev. B 86 224504

[29] Clem J R, Mawatari Y, Berdiyorov G R and Peeters F M 2012 Phys. Rev. B 85144511

[30] Berdiyorov G R, Milošević M V, Latimer M L, Xiao Z L, Kwok W K and Peeters F M 2012 Phys. Rev. Lett. 109 057004

[31] Reichhardt C, Olson C J and Nori F 1998 Phys. Rev. B 586534 